\title{
Observation of NPG VLF Transmissions at Tracy, California During Path Equinox
}

\author{
G. B. Carpenter and A. L. Whitson \\ Stanford Research Institute, Menlo Park, Calif.
}

(Received July 27, 1964; revised October 23, 1964)

\begin{abstract}
Phase and amplitude of five NPG wavefront components were recorded at Tracy, California in March 1964 at a time when the sunrise and sunset lines were almost paralled with the propagation path. Large rapid phase and amplitude variations were associated with sunrise; and at sunset, exactly one full cycle of phase was accumulated on 18 of 20 days of recording. Summation calculations based on simplified ray theory were able to explain, at least qualitatively, most of the gross features of the data. However, phase accumulation on this particular path seems to require an ionospheric model in which partical reflections can be received simultaneously from two heights during the illumination transition.
\end{abstract}

\section{Introduction}

For propagation paths oriented within $\pm 23.5^{\circ}$ of true north there are two periods each year when sunrise or sunset occurs almost simultaneously over the whole path. (The simultaneity is only approximate at ionospheric heights, since the illumination transition no longer follows a great circle projection on the earth's surface.) These periods, which we shall call path equinoxes, offer an excellent opportunity to investigate the rapid and sometimes drastic changes of propagation associated with sunrise or sunset. Having a more complete knowledge of these transition effects, one can perhaps infer corresponding ionospheric changes consistent with the ground observations. With this possibility in mind, $24.0 \mathrm{kHz}$ transmissions from the U.S. Navy VLF transmitter NPG at Jim Creek, Washington $\left(48^{\circ} 12^{\prime} \mathrm{N}, 121^{\circ} 55^{\prime} \mathrm{W}\right)$ were monitored at Tracy, Calif. $\left(37^{\circ} 47^{\prime} \mathrm{N}, 121^{\circ} 24.5^{\prime} \mathrm{W}\right)$ during the equinox period of March 1964. The length of the path was short enough $(1169 \mathrm{~km})$ that even at ionospheric heights the transition was almost simultaneous (within 1 or $2 \mathrm{~min}$ ) over the whole path on the day of the equinox.

Electromagnetic wavefront measuring apparatus employing parallel-plate and large single-turn loop antennas [Parks et al., 1964] was used to record the vertical $\left(E_{v}\right)$ and forward $\left(E_{\theta}\right)$ electric fields and the vertical $\left(H_{v}\right)$, forward $\left(H_{\theta}\right)$, and transverse $\left(H_{\varphi}\right)$ magnetic fields of NPG wavefront at Tracy, Calif. Each field component was detected coherently employing a quadrature technique to obtain rapid response phase and amplitude data. Two quadrature components

1 The work in this paper was supported in part by the U.S. Navy, Bureau of Ships, under Contract NObsr 85271. were obtained for each field component and recorded on chart paper. The two quadrature components were $A \sin \psi$ and $A \cos \psi$, where $A$ is the signal amplitude and $\psi$ is the angular difference between the received signal and a local reference oscillator. The system bandwidth including the recorder was about $70 \mathrm{~Hz}$. The chart speed used was slow and provided a time resolution of about $30 \mathrm{sec}$ (considered adequate for the rate of change expected).

The equinox measurements began 13 March, approximately the same day as the path sunrise equinox, and terminated 1 April 1964. The path sunset equinox occurred about 4 March, but, when measurements began 13 March, the sunset times at the two path end points differed by only $5 \mathrm{~min}$. Two components, $H_{\varphi}$ and $H_{\theta}$, were recorded continuously during this period, while equipment difficulties interrupted $H_{v}, E_{\theta}$, and $E_{v}$ recordings for several days. Two recording channels were utilized beginning 21 March to record the vertical electric field $\left(E_{v}\right)$ component of $18.0 \mathrm{kHz}$ NBA transmissions from Balboa, C.Z.

\section{Experimental Data}

\subsection{Phase Accumulation}

One of the most interesting, and perhaps unexpected, results of the experiment was the abnormal diurnal phase behavior of four of the five recorded wavefront components. On 18 of the 20 experimental days, phase of the $H_{\varphi}, H_{v}, E_{\theta}$, and $E_{v}$ components advanced at sunset and apparently accumulated exactly one full cycle of total phase at that time. The remainder of the diurnal variation was apparently normal when compared with variation on the two days in which phase retarded at sunset (23 March and 1 April). 


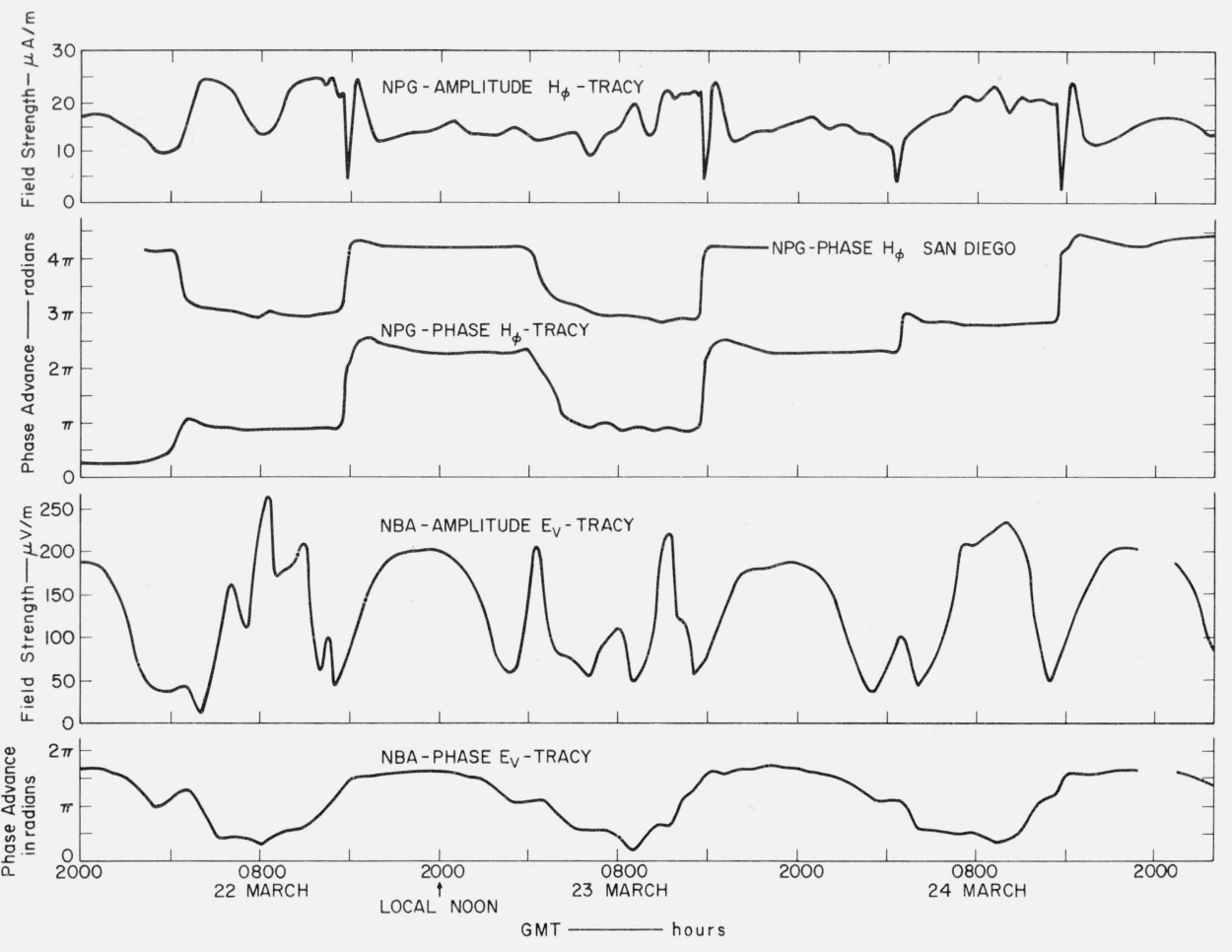

FIGURE 1. Phase and amplitude of NPG and NBA.

Three consecutive days of phase and amplitude data for the $H_{\varphi}$ component of NPG are shown in figure 1 to illustrate the phase accumulation phenomena. Phase accumulated on 22 and 24 March, but not on the 23rd. During this same time interval, the $E_{v}$ component of NBA was monitored and compared with the same frequency standard. These data, also included in figure 1 , do not show phase accumulation. The frequencystandard drift curve derived from the NBA data when corrected for frequency was almost coincident with that obtained from the NPG data assuming exactly one full cycle accumulation per day on days with advance at sunset. The slight discrepancies in the two curves are thought to be introduced by different drift rates of the two transmitting oscillators. The third phase plot in figure 1 is the $H_{\varphi}$ component of NPG recorded at San Diego [Westfall, NEL San Diego-private communication]. The phase variation at San Diego (1780 $\mathrm{km})$ does not show accumulation on either 22 or 23 March, with the only apparent difference in curves for the two days being the more rapid rate of retardation at sunset on the 22nd. The diurnal phase behavior at Tracy and San Diego is similar on 23 March, with the day-to-night phase variation being greater at Tracy.

Phase and amplitude of five components of the NPG wave front are plotted versus time (from local noon to local noon) in figures 2 and 3 on two typical days of the equinox period. The data for 20 March represent a day with an anomalous phase advance at sunset, and data for 1 April show the variation on a day with phase retardation at sunset. The zero phase reference of each signal component has been adjusted for receiver shift.

It is evident from figures 2 and 3 that the diurnal variation of four of the components, $H_{\varphi}, H_{v}, E_{\theta}, E_{v}$, is quite similar. All have a somewhat larger and more erratic amplitude at night than during the day and phase either accumulates on all or none. During the first half of the night on days with no accumulation (fig. 3 , phase shows a gradual variation and is stable during the latter half of the night. Phase is stable throughout the entire night on days with accumulation (fig. 2). Prior to sunset, phase shows a gradual advance on days with accumulation (fig. 2) that is essentially not present on days with no accumulation (fig. 3).

The $H_{\varphi}$ phase and amplitude data shown in figures 2 and 3 are shown in phasor form parametric in time in figure 4. The phasor on both days is advancing between 0100 and 0200 GMT. On 1 April, the phasor suddenly reverses direction at 0200 GMT and phase retards at sunset. On 20 March, the phasor changes direction at 0200 GMT but continues to advance in phase, resulting in advance at sunset. The signal was not lost at any time on either day with the system bandwidth used $(70 \mathrm{~Hz})$.

Amplitude of $H_{\theta}$ is much stronger and much more erratic at night than during the day. The diurnal 

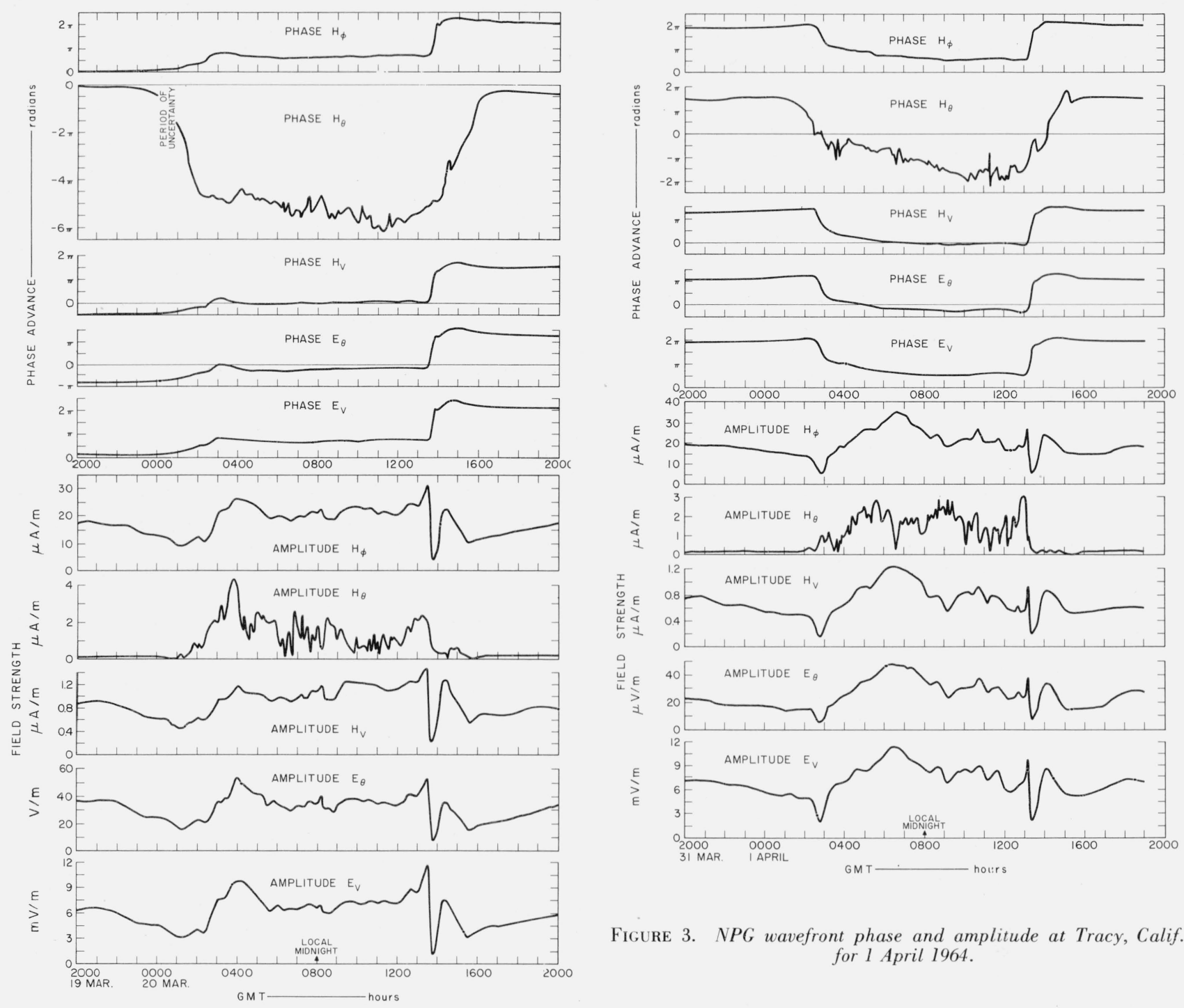

Figure 3. NPG wavefront phase and amplitude at Tracy, Calif. for 1 April 1964.

FigURE 2. NPG wavefront phase and amplitude at Tracy, Calif. for 20 March 1964.
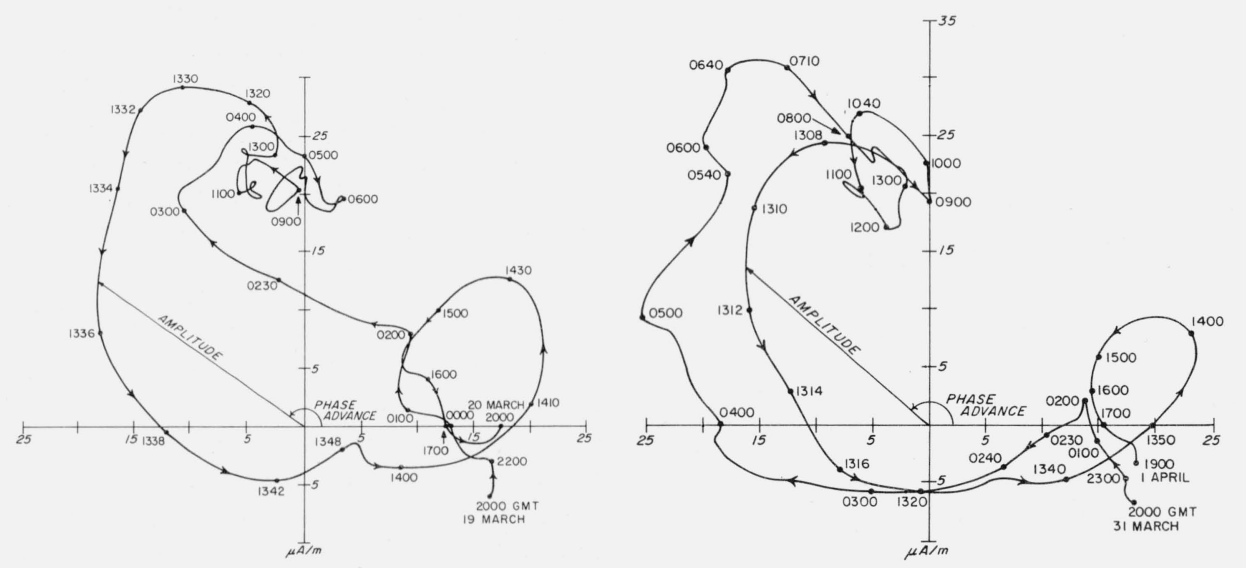

Figure 4. Phasor plot of $\mathrm{H}_{\varphi}$ component of NPG at Tracy, Calif. for 20 March and 1 April 1964. 
phase change of $H_{\theta}$ was about 2.5 cycles on all days except 1 April. On 1 April the $H_{\theta}$ phase change was about 1.5 cycles; however, this smaller change apparently is not necessarily associated with days on which phase of the other four components does not accumulate. Because of full cycle uncertainties during low or zero signal level, accumulation may or may not be present on $H_{\theta}$.

\subsection{Sunrise-Sunset Onset}

The sunrise amplitude and phase behavior of $H_{\varphi}$, $H_{v}, E_{v}$, and $E_{\theta}$ were very repeatable on all days observed. Phase showed a very rapid advance of 0.67 cycles and amplitude exhibited a series of maximums and minimums. Times of the first maximum, first minimum, and second amplitude maximum of the $H_{\varphi}$ component at sunrise are shown in figure 5 for the entire recording period. Sunrise times in the ionosphere can be estimated only crudely for comparison with the experimental data. Such calculations often assume that light rays of a wave length responsible for ionization can not reach the ionosphere by first passing through the dense lower atmosphere. The depth to which rays may penetrate is somewhat controversial and the sunrise times shown in figure 5 for 70 and $90 \mathrm{~km}$ above the two path endpoints were obtained for rays arbitrarily tangent to a sphere $30 \mathrm{~km}$ above the earth's surface. These times are about $11 \mathrm{~min}$ later than for rays tangent to the earth's surface.

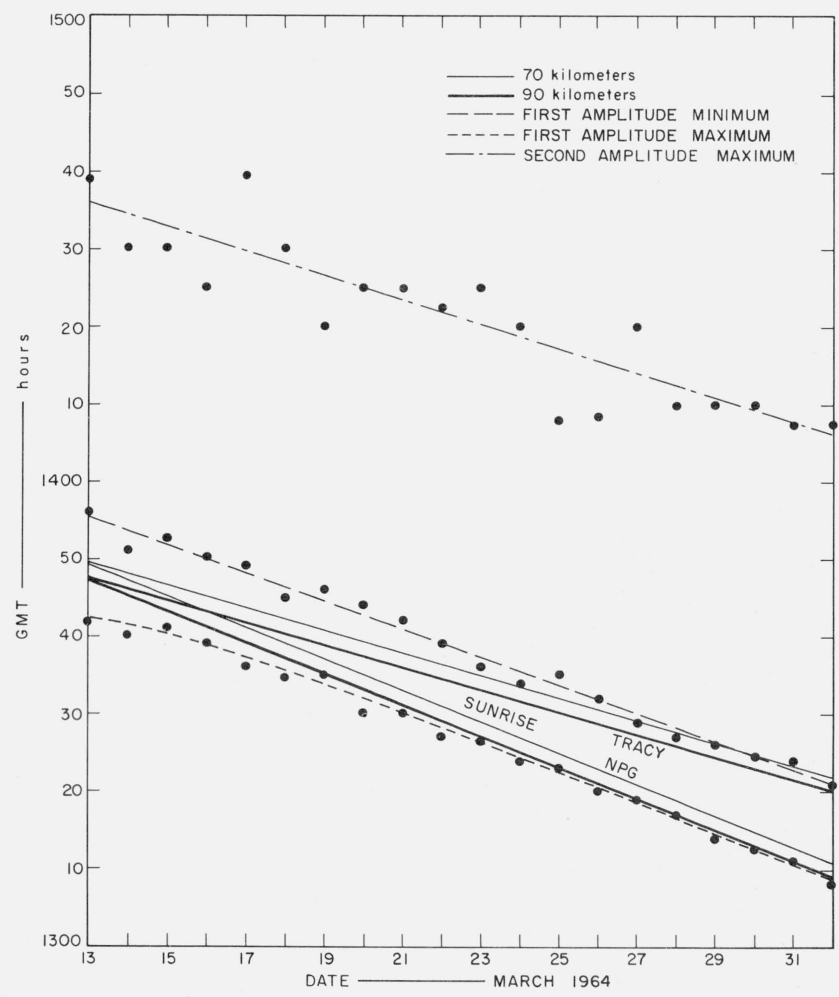

FIGURE 5. Comparison of predicted ionospheric sunrise times with sunrise features of the data.
On 13 March (sunrise equinox), the initial amplitude peak occurs about 5 minutes before the earliest 90 $\mathrm{km}$ sunrise time shown in figure 5 , but by 1 April the times are coincident. The earliest sunrise effects are often observed several minutes before the initial amplitude peak, and consequently, as much as 8 to $10 \mathrm{~min}$ before our earliest predicted sunrise. The data suggest that either sunrise at heights greater than $90 \mathrm{~km}$ influences VLF propagation, or that the effective height of ray tangency is less than $30 \mathrm{~km}$. An alternative and interesting possibility is that the sunrise height transition influences the data while the transition is still some distance from the great circle path.

Predicted optical sunset times along the path were not as well correlated with the time of sunset features of the data as was the case at sunrise. The earliest feature, a "corner" on the phasor plots at about 0200 GMT (see fig. 4), was 10 to 20 min earlier than even the ground sunset. One can only suggest that the decrease in the sun's ionizing flux at high zenith angles before the sun reaches the horizon is responsible.

\section{Discussion and Tentative Explanation of Data}

\subsection{Calculation of Expected Field}

Two methods are generally accepted for predicting signal parameters for a particular VLF transmitterreceiver combination. One method, based on geometrical ray optics, proceeds by summing the contributions of a multiplicity of rays reflected between the earth and ionosphere. This method is particularly useful at short ranges where the higher order reflected rays are of low amplitude, and only a few need be considered. The second method, based on mode theory, consider the earth and ionosphere as boundaries of a spherical waveguide in which certain characteristic modes may propagate. For medium and long paths the higher order modes are heavily attenuated so that it is usually only necessary to sum one or two modes. Volland [1961] has shown the two types of calculations to give approximately the same results in the transition region. Both these methods suffer the difficulty of choosing correct ionospheric models and parameters to make actual numerical calculations.

The $1169 \mathrm{~km}$ separation between NPG and Tracy places this path in the transition region where at least three terms of either the ray or mode series would be required to approximate the signal. For ray theory, this would involve a groundwave and first-, second-, and possibly third-hop rays. Regarding mode theory, the first and second, and probably the third and fourth, modes would be involved during certain periods of the day.

In order to get a rough quantitative estimate of signal to be expected at Tracy, the groundwave and first- and second-hop rays were summed for a number of ionospheric conditions. (Choice of ray theory was prompted by the general lack of tabulated or plotted data for modes of an order greater than one and two.) 
Using the ray summation given by Wait [1962] and ray optics parameters from Wait and Murphy [1957], the expected values of phase and amplitude obtained by vector summation of the groundwave and first- and second-hop reflected rays are as shown in figure 6 . These values are plotted as a function of an assumed reflection height 60 to $100 \mathrm{~km}$ for three specific values of $\omega_{\tau}$, where $\omega_{\tau}$ is defined by Wait [1962] as

$$
\omega_{\tau}=\omega_{0}^{2}\left[\nu^{2}+\omega_{L}^{2}\right]^{-1 / 2},
$$

and $\omega_{0}=$ plasma frequency

$\nu=$ collision frequency

$\omega_{L}=$ gyrofrequency.

The three values of $\omega_{\tau}\left(8 \times 10^{4}, 2 \times 10^{5}\right.$, and $\left.5 \times 10^{5}\right)$ were selected to encompass the value $2 \times 10^{5}$ considered by Wait [1962] to be most representative of actual reflection. The absolute scale of the amplitude plots assumes a radiated power of $200 \mathrm{~kW}$.

The shapes of the calculated amplitude and phase curves are quite similar for all three values of $\omega_{\tau}$, with only small shifts in the magnitude and position of amplitude maximums and minimums and of the phase plateaus. The first-hop ray remains dominant for reflection at all heights, being always about twice the sum of all the other field components. Amplitude maximums or minimums occur when the second-hop ray is nearly in or our of phase with the first-hop ray. For example, when $\omega_{\tau}=2 \times 10^{5}$, amplitude is maximum for reflection heights of 60,80 , and $92.5 \mathrm{~km}$ and minimum for reflection heights of 71 and $86 \mathrm{~km}$. The magnitude of the maximums is greater when the ground wave is nearly in phase with the first- and second-hop rays, and the minimums are deeper when the groundwave and second-hop ray are nearly in phase and both are out of phase with the first-hop ray. None of these conditions were achieved exactly on any of the three plots in figure 6 . The dominance of the first-hop ray is particularly important for its influence on the phase behavior. The second-hop ray contributes only a small superimposed oscillation to the phase pattern and the predicted rate of phase change is greatest during the amplitude maximums and least during the amplitude minimums.

\subsection{Comparison of Calculated and Experimental Data}

The calculated amplitude data must provide for two maximums and two minimums at sunrise and a general increase at sunset. The calculated phase must provide for a total advance of about $1.6 \pi$ radians at sunrise and the possibility of either a retardation or an advance at sunset. The following discussion will be related to the curves for $\omega_{\tau}=2 \times 10^{5}$ (see fig. 6), although discussion for either of the other two sets of curves would be similar.

Sunrise variations can be roughly approximated by a reduction in reflection height, over a period of about an hour, from a nighttime level of $95 \mathrm{~km}$ to a daytime level of $70 \mathrm{~km}$. Amplitude maximums are predicted when the reflection height reaches 92.5 and $80 \mathrm{~km}$,

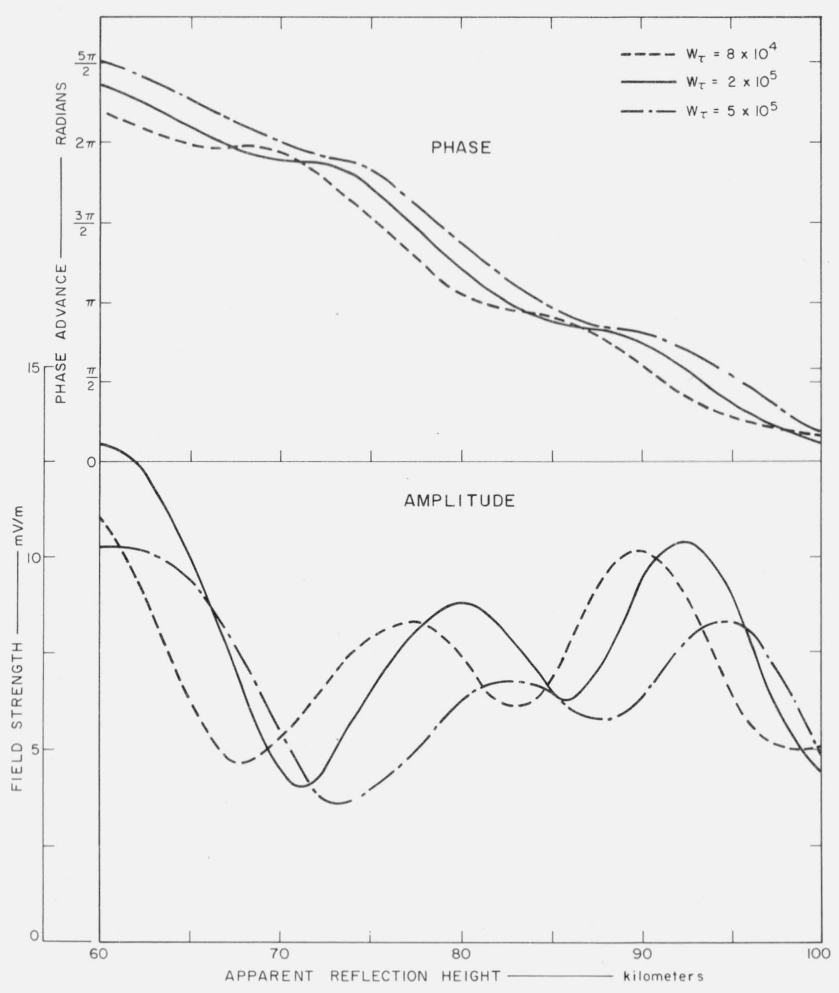

Figure 6. Predicted phase and amplitude of NPG vertical electric field at Tracy as a function of apparent reflection height.

and amplitude minimums when the height reaches 86 and $71 \mathrm{~km}$. The depth of the first amplitude minimum observed in the experimental data is much greater than would be predicted by the calculated curves. The total predicted phase change is adequate, but the actual proportion of phase advance occurring by the time the first amplitude minimum is reached (about $5 / 6$ of the total) is much greater than would be expected from the calculated curves.

The signal variation at sunset is crudely approximated by a return of reflection height from 70 to 95 $\mathrm{km}$. The amplitude minimum expected when the reflection height reaches $86 \mathrm{~km}$ is usually only apparent in the experimental data as a reduction in the rate of amplitude increase. The predicted sunset phase variation approximates the experimental data on the two days when phase retards, but is in no way able to explain a phase advance on the other days. The experimental data indicate that the sunrise and sunset variations are not reciprocal and that the sunset variation is more gradual because of ionospheric relaxation time. The very limited theory that is used here obviously cannot include such variations.

As discussed above, the sunrise and normal sunset variations seem to be explained, at least qualitatively, by the calculated data. The degree of correlation could perhaps be improved by including small changes of $\omega_{\tau}$, along with the change in reflection height $\left(\omega_{\tau}\right.$ also depends on latitude because of change in the 
earth's magnetic field strength). The daytime signal variations do not seem as easily explained in terms of a changing reflection height. Amplitude increases gradually from a low after sunrise to a maximum near local noon to a low at sunset. The amplitude change is accompanied by little, if any, change of phase. The calculated curves suggest that even though the reflection height is held constant, amplitude can be made to increase with little change of phase by varying the value of $\omega_{\tau}$.

The two main discrepancies between the calculated and experimental data are the great depth of the first sunrise amplitude minimum and the phase accumulation at sunset. Both of these features seem to indicate that either the relative magnitudes of the signal components were not as expected, or that some factor or contribution was present that is not considered by the simple theory. The transition periods during path equinox are particularly susceptible to these unusual effects as the ionospheric reflection height changes over the whole path at its maximum rate.

Phase accumulation seems to require that the received signal be separable into two groups of contributions, where the vector sum of one group is larger than the vector sum of the other during one part of the day and smaller during another. If the phases of the two vectors are appropriately related and change at different rates, their vector sum can encircle the origin and accumulate or lose exactly one full cycle per day, although none of the individual contributions do so. Simple ray theory predicts that such conditions can be met for a VLF path of about $600 \mathrm{~km}$ where the ground wave and first-hop ray are nearly equal and again at roughly $2500 \mathrm{~km}$ where the first- and second-hop rays are nearly equal [Volland, 1964].

For a path of $1169 \mathrm{~km}$ one would not normally expect to observe anomalous phase behavior since the first-hop ray predominates at this distance.

For anomalous phase behavior (accumulation) on this path, the effective magnitude of the first-hop ray must be reduced to a level comparable with the vector sum of groundwave and second-hop ray and the phases of the various components must be appropriately related. The small resultant vector, so obtained, is capable of rotating about the origin in either direction depending on the relative magnitudes of the components. The great depth of the sunrise amplitude minimum is consistent with this explanation. (The fact that anomalous phase variation is not observed at sunrise may merely mean that the phases of the components are not appropriately related.) The difficulty with the above explanation is that the signal level at sunset never approaches what might be considered a low value, either during normal phase retardation or anomalous phase advance. Reduction in magnitude of the first-hop ray by a factor of about ten would be necessary to permit both the observed signal strength and anomalous phase behavior. Such a large reduction cannot easily be explained with the simple theory.

The more involved theory probably requires a more realistic ionospheric model than used in the present calculations. Since there are three major ionizing agencies-solar Lyman $\alpha$, solar $\mathrm{x}$ rays, and cosmic rays-and several minor ones, the structure of the $\mathrm{D}$ region is complicated (see review papers by Reid [1964] and Wright [1964]). One manifestation of this more complicated stratified model is the proposed partial reflections from more than one level used by a number of workers to explain certain features of their data [Ellyett and Watts, 1959; Gossard and Paulson, 1964]. Wait and Walters [1963] have indicated that partial reflections are possible from perturbed exponential ionospheric models. Gossard [private communication] has shown that for vertical incidence a small perturbation to an exponential ionosphere can give reflections from two heights. For the NPG-Tracy path, simultaneous partial reflection from two heights during some portion of the sunset transition could cause two first-hop ray components that interfere with each other in such a manner that their resultant is significantly less than the sum of the groundwave and second-hop rays. As noted earlier, this seems to be a requirement for the phase accumulation actually observed in the data.

Basically, the sunset transition process would seem to involve a gradual decrease in magnitude of the ray component partially reflected from the lower level (say the daytime ray) while the ray component partially reflected from the higher level (say the nighttime ray) increases in magnitude. The interchange in relative magnitudes is probably also accompanied by some change of the two phases. The resultant of the two components can have a wide range of variations depending on the two reflection heights and the manner in which the amplitudes and phases of the components vary with time. It is probably significant that for a path of $1169 \mathrm{~km}$ ray optics predict a $180^{\circ}$ difference in phase between first-hop rays partially reflected from $70-72 \mathrm{~km}$ and 88-92 km heights, which are approximately the daytime and nighttime reflection levels. At some time during the transition the resultant of the two antiphase first-hop components could be quite small, allowing phase accumulation.

Although the model with partial reflections seems capable of altering the relative magnitudes of the various signal components to produce phase accumulation, it is difficult to assign actual ray magnitudes and phases as a function of time that will satisfy all the experimental data. It is particularly difficult to identify the physical difference in the reflection region(s) that cause the relationship of the various signal components to be shifted sufficiently from one sunset to the next to allow phase accumulation at one and phase retardation at the other. Data obtained in August 1963 on NPG received at Tracy [Parks et al., 1964] did not indicate any anomalous phase behavior. One can only suggest that sunset at the first-hop reflection region must be nearly simultaneous with sunset at the two second-hop reflection regions to cause a phase accumulation.

The preceding discussion has dealt primarily with the vertical electric field component. Simplified ray calculations for the other five wave front com- 
ponents could have been carried out in a similar manner. Comparison of the equations for $E_{v}, E_{\theta}$, and $H_{\varphi}$ confirm the similar diurnal variation that was actually observed for each. Because of complex ratios of reflection coefficients, the diurnal variation of $H_{v}$, and $H_{\theta}$ would be expected to be different from those above. Of the two latter components recorded, only the $H_{\theta}$ component was noticeably different. (Perhaps because of its small magnitude, measurement of $H_{v}$ may have been contaminated by the strong $H_{\varphi}$ component of the signal.)

The rather unusual behavior of $H_{\theta}$ can be partially explained by the simple theory used in the previous calculations. The main contributions to the $H_{\theta}$ signal are the ${ }_{\|} R_{\perp}$ reflection contribution of the first-hop ray and the ${ }_{\|} R_{\|} R_{\|\|} R_{\perp}$ and ${ }_{\|} R_{\perp} R_{\perp \perp} R_{\perp}$ reflection contributions of the second-hop ray. During the day, all these contributions are small and the resultant signal is small. At night, both the first-hop and second-hop contributions to $H_{\theta}$ are larger and, because of the angle-of-incidence dependence of the convergence coefficients, the two are nearly equal. The large amplitude of $H_{\theta}$ usually observed after sunset and again before sunrise is perhaps an in-phase addition of these two contributions. The erratic behavior of $H_{\theta}$ during the night is perhaps produced by the approximately equal first- and second-hop contributions being more nearly out of phase. The diurnal change of $H_{\theta}$ phase can be expected to differ from that of the normal components $\left(H_{\varphi}, E_{v}\right.$, and $\left.E_{\theta}\right)$ because of different reflection parameters. However, the large observed $H_{\theta}$ diurnal phase change and its day-to-day variation tend to indicate that during sunlight transitions phase may be gaining and losing cycles due to repeated encirclement of the origin by the resultant of two nearly equal contributions.

\section{Summary}

Wave front measurements of NPG transmissions at Tracy, Calif. during the path equinox show that sunrise effects appear to begin as much as $10 \mathrm{~min}$ before the sun's rays (tangent to a $30 \mathrm{~km}$ high ozonosphere) reach the ionosphere $90 \mathrm{~km}$ above the propagation path. During the sunrise transition, amplitude decreases of 15-35 $\mathrm{dB}$ and phase advances of about $3 / 4$ cycle occur in as short a time as $12 \mathrm{~min}$. The initial effect of the sunset transition occurs 10 to 20 min earlier than even the ground sunset along the propagation path and suggests that the earliest sunset variation in the ionosphere occurs at high zenith angles before the sun reaches the horizon.
During the sunset transition, phase accumulation of exactly one cycle per day was observed on 18 of 20 days. The anomalous phase variations appear to be explainable if an ionospheric model in which simultaneous partial reflection from two levels is assumed during the sunset transition.

Interpretation of the observed sunrise-sunset and phase accumulation phenomena is not possible using available simple theory. One can only infer what may be the cause of observed data. A more complete set of measurements to completely define the many variables that influence VLF propagation is required so that interpretation using the most complex and complete propagation theory available can be applied to gain an understanding of the problem.

We are grateful to G. H. Price for his very helpful suggestions and discussions related to the data interpretation.

\section{References}

Ellyett, C., and J. M. Watts (Sept.-Oct. 1959), Stratification in the lower ionsophere, J. Res. NBS, 63D (Radio Prop), No. 2, 117-134.

Gossard, E. E., and M. R. Paulson (March 1964), An interpretation of rapid changes in the phase of horizontally polarized VLF waves recorded at night over a short path in the southwestern United States, Radio Sci. J. Res. NBS/USNC-URSI 68D, No. 3, 265-273.

Parks, G. S. Jr., G. H. Price, A. L. Whitson, and H. W. Parker (March 1964), Measurements of VLF wave-front components over long paths, Final Report Contract NObsr 85271 (FBM), Stanford Research Institute, Menlo Park, California.

Reid, G. C. (May 1964), Physical processes in the D region of the ionosphere, Review of Geophysics, 2, 311-333.

Volland, H. (July-Aug. 1961), Comparison between mode theory and ray theory of VLF propagation, J. Res. NBS, 65D (Radio Prop), No. 4, 357-561.

Volland, Hans (Feb. 1964), Diurnal phase variations of VLF waves at medium distances, Radio Sci. J. Res. NBS/USNC-URSI, 68D, No. 2, 225-238.

Wait, J. R., and A. Murphy (June 1957), The geometrical optics of VLF sky wave propagation, Proc. IRE 45, 754-760.

Wait, J. R. (July 1962), Introduction to the theory of VLF propagation, Proc. IRE 50, 1624-1647.

Wait, J. R., and L. C. Walters (Oct. 1963), Reflection of VLF radio waves from an inhomogeneous ionosphere. Part II. Perturbed exponential model, J. Res. NBS 67D (Radio Prop), No. 5, 519-523.

Wright, J. W. (May 1964), Electron distribution in the ionosphere, Radio Sci. J. Res. NBS/USNC-URSI, 68D, No. 5, 589-594.

(Paper 69D4-500) 